\title{
Diagnostic Utility of Cell Population Data (CPD) In Sepsis Using Automated Hematology Analysers
}

\author{
Shalini.P ${ }^{1}$, Purnima S Rao ${ }^{1 *}$, Sneha Rao A R ${ }^{1}$, Manjula Anil ${ }^{2}$, Athira Benny ${ }^{1}$ and Sandhya $I^{1}$ \\ ${ }^{1}$ Department of Pathology, A.J Institute of Medical Sciences and Research Centre, Mangalore, India \\ ${ }^{2}$ Department of Community Medicine, A.J Institute of Medical Sciences and Research Centre, Mangalore, India
}

\begin{abstract}
Background: Sepsis still remains an important cause of morbidity and mortality posing a diagnostic challenge to clinicians across the globe. Lack of a single specific and sensitive test paved the way to many researches. Our study evaluated clinical significance of cell population data (CPD) as biomarkers in sepsis. CPD assesses morphological and functional characteristics of leucocytes using automated hematology analyzer.
\end{abstract}

Methods: The study population consisted of 100 healthy subjects and 100 clinically suspected cases of sepsis with quick sequential organ failure assessment (qSOFA) score $>2$. The various cell population data (CPD) were collected using automated hematology analyzer, Sysmex XN1000 during a span of 6 months from January to June 2018 in a tertiary care center and the results were statistically analyzed using $\mathrm{Z}$ test.

Result: The WBC count and CPD parameters were assessed. Except for neutrophil, monocyte cell size (NE-FSC, MO-Z) and lymphocyte fluorescence intensity $(\mathrm{LY}-\mathrm{Y})$ all other CPD parameters show significant difference $(\mathrm{p}<0.001)$ in sepsis group compared to healthy controls.

Conclusion: This study suggested utility of CPD parameters which provide details of size and internal structure of leucocytes to be considered as an important biomarker for diagnosis and management of sepsis.

Keywords: Sepsis, CPD, automated hematology analyzer

\section{Introduction}

Centre for disease control and diagnostics (CDC) defines sepsis as generalized, overwhelming and life-threatening response to infection. Sepsis is a leading cause of morbidity and mortality amounting to a global burden of 30 million cases annually. The timely diagnosis and monitoring of patients with sepsis have major impact in clinical outcome of the patients. ${ }^{1}$

There is no single specific test in diagnosis of sepsis. Biomarkers like lactic acid, procalcitonin, $C$ reactive protein (CRP), immature granulocytes and delta neutrophil index are widely used in sepsis, but their major drawbacks are cost ineffectiveness, requirement of technical expertise and less diagnostic sensitivity. ${ }^{1,2}$ Lack of a sensitive screening test for early detection and a specific test to confirm the diagnosis paved the way to many researches. Therefore, novel parameters of leucocytes and its differentials is gaining importance as a screening test. Modern automated hematology analyzers combine flow cytometry and fluorescence to assess individual cells in an accurate, fast and cost-effective manner. Cell granularity, cell volume, shape, nucleic acid and protein content of cells is assessed on the basis of side scatter, forward scatter and fluorescent light intensity. This yields a complete blood count and cell population data (CPD) which provides quantitative and qualitative information about various leucocyte differentials. Primary assessment of cell population data by various studies showed its utility in myelodysplastic syndrome and sepsis ${ }^{3,4,5}$

This study aimed at assessing the clinical relevance of CPD parameters in sepsis patients when compared with normal healthy controls.

\section{Materials and Methods}

This study was conducted in a tertiary care center for a period of 6 months from January 2018 to June 2018. The study population comprises of 100 clinically suspected cases of sepsis with qSOFA (Quick sequential organ failure assessment) score greater than 2. Exclusion criteria being age less than 18, hematologic disorders and liver diseases. The study also included 100 ostensibly healthy controls.

The blood samples were collected under complete aseptic precautions in EDTA vacutainers and were analyzed using automated hematology analyzer Sysmex XN1000 within 2 hours of sample collection.

The parameters evaluated included total blood count and CPD of neutrophils, lymphocytes and monocytes on WDF channel. The 3 axis of WDF channels uses optical signals 
along $\mathrm{X}$ axis (side scatter) to assess the internal complexity, fluorescence along $\mathrm{Y}$ axis for the nucleic acid content and the forward scatter along the $\mathrm{Z}$ axis was in accordance with the cell size.

The parameters on $\mathrm{X}$ axis include neutrophils cell complexity (NE-SSC), lymphocytes cell complexity (LY-X), monocytes cells complexity (MO-X), neutrophils complexity and width of dispersion (NE-WX), lymphocytes complexity and width of dispersion (LYWX) and monocytes complexity and width of dispersion $(\mathrm{MO}-\mathrm{WX})$. The parameters reported on the Y-axis include neutrophils fluorescence intensity (NE-SFL), lymphocytes fluorescence intensity (LY-Y), monocytes fluorescence intensity (MO-Y), neutrophils fluorescence intensity and the width of dispersion (NE-WY), lymphocytes fluorescence intensity and the width of dispersion (LY-WY) and monocyte fluorescent intensity and width of dispersion (MO-WY). The following parameters were reported on the Z-axis, neutrophils cell size (NE-FSC), lymphocytes cell size (LY-Z), monocytes cell size (MO-Z), neutrophils cell size and the width of dispersion (NE-WZ), lymphocytes cell size and the width of dispersion (LY-WZ) and monocytes cell size and the width of dispersion (MO-WZ). .

The distribution of values of CPD parameters were assessed between the 2 groups. The mean and standard deviation of each class was calculated and further statistical analysis to assess significant difference between two groups was done using $\mathrm{Z}$ test. The non-parametric test was used for LY-Y and NE-FSC. The resulting p-value $<0.05$ is considered statistically significant.

As the investigations were done on pre-existing samples, ethical clearance and informed consent was not required.

\section{Result}

The total WBC count and 18 CPD parameters were compared between 100 normal healthy controls and 100 patients with qSOFA score $>2$.
The total WBC count among sepsis and control showed a mean of 18.10 and 6.96 respectively with a statistically very high significant difference with $\mathrm{p}<0.001$. (table1)

Among the CPD parameters evaluated, 15 parameters (83\%) show statistically high significant difference.

All the neutrophilic parameters showed very high significance between the 2 groups with $p$ value $<0.001$ with exception of NE-FSC which was statistically insignificant with $\mathrm{p}$ value $>0.05$ (table 1). This indicates the increase amount of cellular DNA and RNA and degree of heterogeneity of neutrophil population with minimal variation in cell size.

The monocyte parameters with exception of MO-Z showed very high significance statistically with $\mathrm{p}$ value $<0.001$. (table1) This indicates increase in amount of cellular DNA and RNA, granules, vacuoles and degree of heterogeneity of monocyte population with minimal variation in cell size.

Among the lymphocyte data, all values were significant statistically especially those reflecting cellular dispersion with $p$ value $<0.001$ with exception of LY-Y parameter with showed a negative correlation with respect to sepsis. (table 1) This indicates increase of granules, vacuoles with heterogeneity of lymphocyte population and minimal change in cellular DNA and RNA

There was no statistical significance found in age and gender among the sepsis group and the control group.

Thus, all the CPD parameters with exception of NE-FSC, MO-Z and LY-Y show statistically high significance in the clinically suspected sepsis cases with qSOFA score $>2$ compared to normal healthy controls.

\section{Discussion}

Sepsis evokes immune response to various pathogens resulting in multiorgan dysfunction. It is a biphasic process consisting of early inflammatory and a later antiinflammatory phase. Hematologic system is one of the first

TABLE : 1

\begin{tabular}{|c|c|c|c|c|c|}
\hline \multirow{2}{*}{ Items } & \multicolumn{2}{|c|}{$\begin{array}{c}\text { Sepsis (N=100) Mean Standard } \\
\text { deviation }\end{array}$} & \multicolumn{2}{c|}{$\begin{array}{c}\text { Healthy controls(N=100) Mean } \\
\text { Standard deviation }\end{array}$} & $\begin{array}{c}\text { P VALUE } \\
\text { WBC }\end{array}$ \\
\hline NE-SFL & 56.11 & 6.95 & 6.96 & 2.94 & $\mathrm{p}<0.001$ \\
\hline NE-FSC & 95.11 & 11.09 & 45.77 & 6.39 & $\mathrm{p}>0.001$ \\
\hline NE-WX & 333.87 & 80.97 & 87.22 & 319.46 & $\mathrm{p}<0.001$ \\
\hline NE-WY & 809.62 & 161.06 & 607.15 & 28.64 & $\mathrm{p}<0.001$ \\
\hline
\end{tabular}




\begin{tabular}{|c|c|c|c|c|c|}
\hline \multirow{2}{*}{$\begin{array}{l}\text { Items } \\
\text { NE-WZ }\end{array}$} & \multicolumn{2}{|c|}{$\begin{array}{c}\text { Sepsis }(\mathbf{N}=100) \text { Mean Standard } \\
\text { deviation }\end{array}$} & \multicolumn{2}{|c|}{$\begin{array}{l}\text { Healthy controls }(\mathrm{N}=100) \text { Mean } \\
\text { Standard deviation }\end{array}$} & \multirow{2}{*}{$\begin{array}{l}\text { P VALUE } \\
p<0.001\end{array}$} \\
\hline & 719.01 & 107.04 & 674.08 & 37.27 & \\
\hline LY-X & 75.75 & 3.86 & 74.49 & 2.2 & $p<0.01$ \\
\hline LY-Y & 122.92 & 577.14 & 66.16 & 5.33 & $p>0.05$ \\
\hline LY-Z & 61.58 & 2.43 & 60.49 & 1.14 & $p<0.001$ \\
\hline LY-WX & 641.66 & 143.43 & 566.47 & 68.03 & $p<0.001$ \\
\hline LY-WY & 1113.71 & 271.50 & 918.10 & 81.60 & $p<0.001$ \\
\hline LY-WZ & 632.38 & 157.20 & 520.3 & 29.60 & $p<0.001$ \\
\hline MO-X & 118.38 & 4.47 & 113.19 & 2.88 & $p<0.001$ \\
\hline MO-Y & 114.64 & 13.70 & 107.88 & 8.24 & $p<0.001$ \\
\hline MO-Z & 69.19 & 3.68 & 68.87 & 2.37 & $p>0.05$ \\
\hline MO-WX & 310.85 & 61.43 & 246.69 & 27.65 & $p<0.001$ \\
\hline MO-WY & 769.64 & 149.35 & 682.35 & 109.07 & $p<0.001$ \\
\hline MO-WZ & 650.15 & 85.512 & 597.50 & 62.41 & $p<0.001$ \\
\hline
\end{tabular}

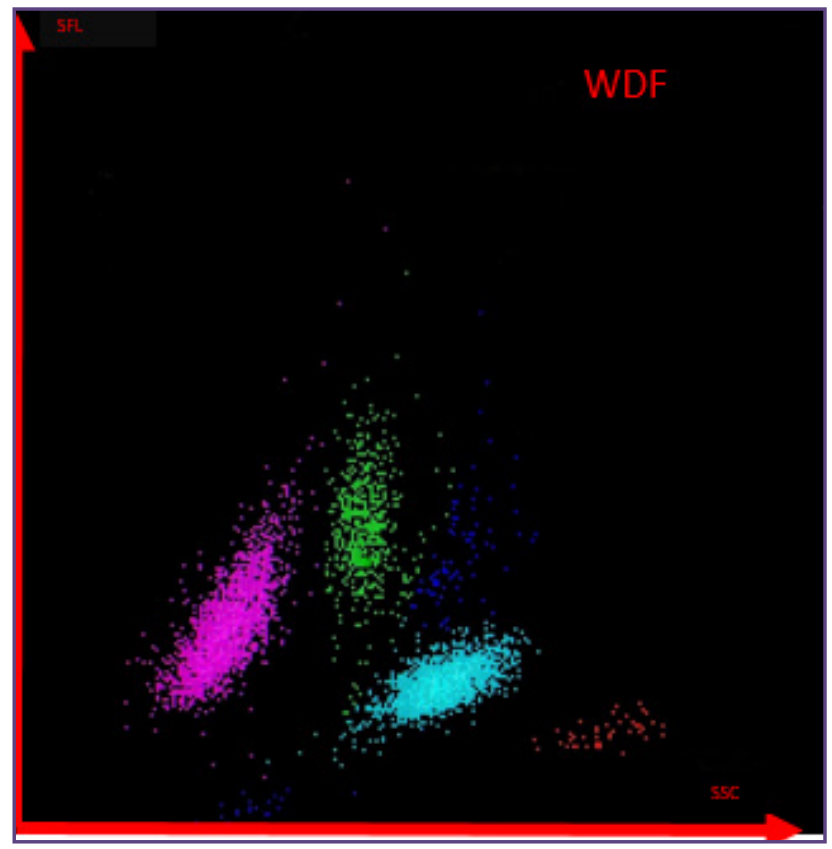

Fig. 1: Scattergram displays groups of lymphocytes, monocytes, eosinophils,basophils + neutrophils and debris

to get affected in sepsis. Innate immune system with its defenders like neutrophils, monocytes/macrophages and NK cells come to action. The cytokines released during the inflammatory process cause the migration of mature and immature forms of leucocytes from the bone marrow pool. Neutrophils are primarily involved in phagocytosis and show presence of toxic granules, residual RNA (Dohle bodies) and cytoplasmic vacuoles. Monocytes by the presence of toll like receptors and by upregulating synthesis and release of cytokines play important role in innate system. Blood lymphocyte dysfunction is also recognized. ${ }^{6,7}$ Thus the quantitative and qualitative assessment of various leucocyte parameters with automated hematology analyzer like Sysmex XN 1000 will aid in the early diagnosis of sepsis, thereby substantially improving the clinical outcome of the patients. Since these parameters are automatically generated it would serve as a rapid and inexpensive tool to the clinicians thereby overcoming the major drawbacks of commercially available biomarkers. ${ }^{6,7,8}$

The Sysmex XN 1000 automated hematology analyzer uses fluorescence flow cytometry to analyze the WBC differential count in combination with forward scatter (FSC), side scatter (SSC) and fluorescence intensity (SFL) by perturbation of cells with a fluorescent dye. Detection of scattered light when a laser beam is emitted to blood cell particles helps us to assess cell size (forward scatter) and cell interior (side scatter). It also measures the intensity of fluorescence emitted to assess degree of blood cell staining (nucleic acid). These parameters when combined with width of dispersion (W) yields series of CPD parameters of neutrophils (NE), lymphocytes (LY) and monocytes (MO). These are represented in the three axes of WBC differential fluorescence (WDF) scattergram (figure1). 
In WDF channel, surfactant causes lysis and dissolution of platelets and RBCs thus avoiding its interference. 4,5 The clinical utility of these parameters in screening sepsis, myelodysplastic syndrome, acute leukemia and lymphoproliferative disorders are still under trial. ${ }^{8} \mathrm{CPD}$ parameters assess morphologic variation in cells in response to various stimuli like infections. The activated cell membranes are more permeable to reagents like fluorescent dye which can bind to organelles and nucleic acids. Thus, CPD parameters evaluate changes in size, shape and nucleic acid content in cells. $.^{9}, 10$

Here we considered the clinical utility of CPD parameters, in which majority with exception of NE-SFC and MO-Z showed significant change in sepsis cases compared to normal healthy controls. This is in accordance with studies done by Urrechaga et al, Park et al which emphasized the utility of various CPD parameters in early diagnosis of sepsis. Urrechaga et al emphasized the importance of NE-SFL and MO-X in sepsis cases and proposed a NEMO (Neutrophilic and monocytic) scoring system for early diagnosis of sepsis based on these parameters. It consists of neutrophil count, lymphocyte count, IG\%, NESFL and MO-X.The score stratifies the patient into mild (score $<3)$, moderate (score 4-5) and severe (score $>6$ ). Though promising, the score needs further work up to include in daily clinical practice. Another study done by Boveda et al on neutrophilic cell population data in acute bacterial infections got a positive correlation with NEWY indicating the increase in nucleic acid content in neutrophils in response to bacterial infections.

LY-Y parameter showed a particular trend in our study with negative correlation in sepsis cases, which was consistent with Buoro et al. However, the major disadvantage of these CPD parameters were less analytical quality specification and harmonization among different instruments and clinical laboratories. ${ }^{11}$ This was rectified to an extent by the study done on quality control assessment and harmonization of CPD parameters by Seghezzi et al. They found minimal imprecision for all CPD parameters except for those reflecting width of dispersion. Though in our study, no imprecision test was performed, combining our statistical analysis with Seghezzi et al we would like to streamline our findings to all CPD parameters with exception NESFC, MO-Z, LY-Y and those reflecting width of dispersion would aid in early diagnosis of sepsis.

\section{Conclusion}

Sepsis still remains a diagnostic dilemma to many clinicians across the globe. Many biomarkers due to their major limitations like availability, diagnostic insufficiency, cost, and high turnover around time makes them counterproductive. This paves the way to newer research parameters which are appropriable and accessible for early diagnosis of sepsis. Automated hematology analyzers using fluorescent flow cytometry yield various CPD parameters which assess quantitative and qualitative changes in individual cells. Thus, with availability of calibration and quality control, CPD parameters are highly advisable in early diagnosis and management of sepsis in near future.

\section{Acknowledgements}

Completion of this undertaking could not have been possible without utmost guidance and support of our departmental head Dr. Muktha R Pai. We would also like to thank entire staff, Department of pathology for their endless support during our study. We are thankful to our families whose unflinching support has been a pillar of strength. Above all, great almighty, the author of knowledge and wisdom for his countless love.

\section{Reference}

1. Buoro S, Seghezzi M, Vavassori M,et al. Clinical significance of cell population data (CPD) on Sysmex XN-9000 in septic patients with or without liver impairment .Ann Transl Med 2016;4:418.

2. Park SH, Park CJ, Lee BR, et al. Sepsis affects most routine and cell population data (CPD) obtained using the Sysmex XN-2000 blood cell analyzer: neutrophil-related CPD NESFL and NE-WY provide useful information for detecting sepsis. Int J Lab Hematol 2015;37:190-8

3. Le Roux G, Vlad A, Eclache V, et al. Routine diagnostic procedures of myelodysplastic syndromes: value of a structural blood cell parameter (NEUT-X) determined by the Sysmex XE-2100. Int J Lab Hematol 2010;32:237-43

4. Urrechaga E, Boveda O, Aguirre U. Role of leucocytes cell population data in the early detection of sepsis. J clin pathol 2017; 0:1-8

5. Buoro S, Mecca T, Azzarà et al. Extended leukocyte differential count and C-reactive protein in septic patients with liver impairment: diagnostic approach to evaluate sepsis in intensive care unit. Ann Transl Med 2015;3(17):244

6. Goyette R E, Key N S, Ely E W. Hematologic changes in sepsis and their therapeutic implications. Seminars in respiratory medicine and critical care 2004;25:646-659

7. Funke A, Berner R, Trailer B, Schmeisser D, Leitis JU, Neimeyer C M..Frequency,natural course, and outcome of neonatal neutropenia.Pediatrics 2000;106:45-51

8. Furundarena JR, Araiz M, Uranga M, et al. The utility of the Sysmex XE-2100 analyzer's NEUT-X and NEUT-Y parameters for detecting neutrophil dysplasia in myelodysplastic syndromes. Int J Lab Hematol 2010;32:360-6

9. Urrechaga E, Boveda O,Aguirre U,Garcia S,Pulido E.Neutrophil cell population data biomarkers for acute bacterial infection..J pathol infect dis 2018;1:1-7 
10. Linssen J, Aderhold S, Nierhaus A, et al. Automation and validation of a rapid method to assess the neutrophil and monocyte activation by routine fluorescence flow cytometry invitro .Cytometry B Clin cytom 2008;74:295-309.

11. Seghezzi M ,Sabrina B, Previtali G.A preliminary proposal for quality control assessment and harmonization of leuckocytes morphology -structural parameters. J med biochem 2018;37:1-13
12. Lippi G, Plebani M. Biomarker research and leading causes of death worldwide: a rather feeble relationship. Clin Chem Lab Med 2013;51:1691-3

13. Chaves F, Tierno B, Xu D. Quantitative determination of neutrophil VCS parameters by the Coulter automated hematology analyzer: new and reliable indicators for acute bacterial infection. Am J Clin Pathol 2005;124:1-5.

14. Vincent JL, Beumier M. Diagnostic and prognostic markers in sepsis. Expert Rev Anti Infect Ther 2013;11:265-75.

*Corresponding author:

Dr. Purnima Rao, Postal Address: 204, Aadheeshwari apartments, Mahamaya templeroad, Fieldstreet, Mangalore-575001 INDIA

Phone: +919480055270

Email: purnimashenoy@gmail.com

Financial or other Competing Interests: None. 